\title{
A Neural Network Model for Image Change Detection Based on Fuzzy Cognitive Maps
}

\author{
Gonzalo Pajares ${ }^{1}$, Alfonso Sánchez-Beato ${ }^{2}$, Jesús M. Cruz ${ }^{3}$, and José J. Ruz ${ }^{3}$ \\ ${ }^{1}$ Dpt. Ingeniería del Software e Inteligencia Artificial, Facultad Informática, Universidad \\ Complutense, 28040 Madrid, Spain \\ pajares@dacya.ucm.es \\ ${ }^{2}$ Dpt. Informática y Automática, E.T.S. Informática UNED, 28040 Madrid, Spain \\ alfonsosanchezbeato@yahoo.es \\ ${ }^{3}$ Dpt. Arquitectura Computadores y Automática, Facultad Informática, Universidad \\ Complutense, 28040 Madrid, Spain \\ \{jmcruz, jjruz\} @dacya.ucm.es
}

\begin{abstract}
This paper outlines a neural network model based on the Fuzzy Cognitive Maps (FCM) framework for solving the automatic image change detection problem. Each pixel in the reference image is assumed to be a node in the network. Each node has associated a fuzzy value, which determines the magnitude of the change. Each fuzzy value is updated by a trade-off between the influences received from the fuzzy values from other neurons and its own fuzzy value. Classical approaches in the literature have been designed assuming that the mutual influences between two nodes are symmetric. The main finding of this paper is the assumption that mutual influences could not be symmetric. This non symmetric relationship can be embedded by the FCM paradigm. The performance of the proposed method is illustrated by comparative analysis against some recent image change detection methods.
\end{abstract}

\section{Introduction}

A major portion of the research efforts of the computer vision community has been directed towards the study of automatic image change detection methods [1]. In [2] we have proposed a Hopfield Neural Network (HNN) model, where the comparative analysis against some existing strategies has been proven favorably. In HNN, given a reference image we build a network of nodes where each pixel in the reference image is a node in the network. The goal is to determine if a pixel has changed based on the corresponding node's value. Each node value is updated iteratively through a tradeoff between two kinds of relations: binary and unary. Binary relations establish the degree of influence that a neuron exercises over other neuron during the iterative process. Unary relations determine the self-influence of each neuron during such process. Binary relations have been used in some well-tested strategies [3,4]. The binary relations in HNN are mapped as symmetric weights, as required by the Hopfield neural network paradigm. This symmetry is also used in [3,4] where an energy function is to be minimized. The minimization is carried out by taking into account 
only the previous system configuration (state) without to resort to historic states. This implies that these approaches follow the Markov Random Fields framework.

The main finding of this paper is the assumption that mutual influences could not be symmetric, based on the hypothesis that different nodes could have different levels of relevance. So, assuming that the node $i$ is more relevant than the node $j$, the influence of $i$ over $j$ should be greater than the influence of $j$ over $i$. The relevance is a concept introduced in this work based on the assumption that the value of a relevant node should remain unchanged during the iterative process. The neurofuzzy FCM paradigm is a suitable strategy to deal with non-symmetric influences under binary relations and also to embed the self-influence under unary relations.

This paper is organized as follows. Section 2 contains the customized FCM approach, where the mapping of mutual influences is obtained. Also the mapping of the relevance associated to each node is computed. The performance of the method is illustrated in section 3, where a comparative analysis against other existing image change detection strategies is carried out. Finally, in section 4, there is a discussion of some related topics.

\section{Fuzzy Cognitive Maps for Image Change Detection}

\subsection{The FCM Framework for Image Change Detection}

FCMs are networks used to create models as collections of concepts and the various causal relations that exist between these concepts [5-11]. The concepts are represented by nodes and the causal relationships by directed arcs between the nodes. Each arc is accompanied by a causal weight that defines the type of causal binary relation between the two nodes. The causal weights $w_{i j}$ take values in the fuzzy causal interval $[-1,+1] ; w_{i j}=0$ indicates no causality. Positive causal relation $\left(w_{i j}>0\right)$ between two concepts $C_{i}$ and $C_{j}$ indicates causal increase: $C_{i}$ increases as $C_{j}$ increases and $C_{i}$ decreases as $C_{j}$ decreases. Negative causal relation $\left(w_{i j}<0\right)$ indicates causal decrease or negative causality: decreases as $C_{j}$ increases and $C_{i}$ increases as $C_{j}$ decreases. In FCMs no feedback from a node to itself is allowed, so $w_{i i}=0$.

The image change detection problem is formulated as follows: given two registered images $I_{1}(x, y), I_{2}(x, y)$ of size $M x N$ of the same area in the scene, taken at different times, the goal is to detect if a pixel, located at $(x, y)$, has changed and the magnitude of the change. With such purpose, we build a network of $n=M x N$ nodes, where each node $i$ represents a pixel location $(x, y)$. We create the $n \times n$ weight matrix $W$, assuming that it can be non-symmetric. At each iteration $k$, the node $i$ in the network has the activation level $A_{i}^{k}$ ranging in $[-1,+1]$. This implies that every node is positively or negatively activated to a certain degree that determines the magnitude of the change at each pixel location, i.e. $A_{i}^{k}=+1$ maximum degree of change and $A_{i}^{k}=-1$ without change. 
In Bruzzone and Prieto [3] and Aach and Kaup [4] an iterative scheme determines if a change has occurred at a given pixel location based on the mapping of data and contextual consistencies between such pixel location and the pixels in its neighbourhood. The contextual consistencies only take into account the previous state without to resort to historic states. This implies that they follow the Markov Random Field (MRF) framework. In Pajares [2], the contribution of the neighbours is also mapped as data and contextual consistencies; but unlike in $[3,4]$, an explicit self-contribution is incorporated. So, the degree of change for each pixel is achieved through a tradeoff between the consistencies provided by the neighbours and its own contribution. This scheme, based on the HNN approach, requires that the binary relations between nodes $i$ and $j$ are symmetric $\left(w_{i j}=w_{j i}\right)$, i.e. $W$ must be symmetric. Although, this method performs favourably, we have verified that the mapping of the contextual consistencies can be still improved. Indeed, we assume that different nodes could have different levels of relevance. In this paper, the relevance is determined as a measure of the strength that a node shows, during the iterative process, against the variations on its activation level. The mutual relevance is embedded in the mapping of the contextual information. This implies that $W$ could not be symmetric; hence the HNN paradigm cannot be applied. Nevertheless, the FCM scheme with certainty neurons, Tsardias and Margaritis [5,6], has the ability to assume this non-symmetry. Additionally, it can embed the unary relations. Indeed, a certainty neuron $i$ has memory capabilities and its activation level at iteration $k+1$ is computed as,

$$
A_{i}^{k+1}=f\left(A_{i}^{k}, S_{i}^{k}\right)=f\left(A_{i}^{k}, \sum_{j=1}^{n} w_{j i} A_{j}^{k}\right)-d_{i} A_{i}^{k}
$$

where $A_{i}^{k}$ is the activation of certainty neuron $i$ at iteration $k, S_{i}^{k}$ is the sum of the weighted influence that certainty neuron $i$ receives at the iteration $k$ from all other neurons, $d_{i} \in[0,1]$ is the decay factor of certainty neuron $i$. This factor determines the fraction of the current activation level that will be subtracted from the new activation level as a result of the neuron's natural intention to get closer to activation level zero. The bigger the decay factor the stronger the decay mechanism. Following Tsadiras and Margaritis [5] the function $f$ is that used in the MYCIN expert system for the aggregation of the certainty factors, defined as follows,

$$
f(x, y)= \begin{cases}x+y(1-x) & \text { if } x, y \geq 0 \\ x+y(1+x) & \text { if } x, y<0 \\ (x+y) /(1-\min (|x|,|y|)) & \text { else }\end{cases}
$$

where $|x|,|y| \leq 1 . A_{i}^{k}$ is always in that interval, but this does not apply for $S_{i}^{k}$, as a concept can be influenced by many concepts and perhaps the sum $\sum_{j=1}^{n} w_{j i} A_{j}^{k}$ can take a value outside the interval $[-1,+1]$. In order to keep $S_{i}^{k}$ within that interval it is passed through the sigmoid function, i.e. $S_{i}^{k}=\tanh \left(S_{i}^{k}\right)$, as suggested by Tsadiras and Margaritis [5]. So, under the FCM framework the binary influences are mapped 
in $S_{i}^{k}$ as data and contextual consistencies, assuming that they could be nonsymmetric; the self-influence is embedded in the $A_{i}^{k}$ memory term. From equation (1), the goal is to compute: $a$ ) the $w_{i j}$ in order to build the weight matrix $W$ and $b$ ) the decay factor.

\subsection{Data and Contextual Consistencies}

The data and contextual consistencies are computed following the model described in Pajares [2] and related references. From the images $I_{1}(x, y), I_{2}(x, y)$ we compute the difference image $D(x, y)=I_{1}(x, y)-I_{2}(x, y)$ assuming that each pixel of $D$ is either with the hypothesis $H_{0}$ (no change) or the hypothesis $H_{1}$ (change). We build a Bayesian model so that each pixel $(x, y)$ in the difference image $D(x, y)$ should be associated to the hypothesis that maximizes the posterior conditional probability, i.e.

$$
H_{s}=\arg \max _{H_{s} \in\left\{H_{0}, H_{1}\right\}}\left\{P\left(H_{s} / D(x, y)\right)\right\}=\arg \max _{H_{s} \in\left\{H_{0}, H_{1}\right\}}\left\{P\left(H_{s}\right) p\left(D(x, y) / H_{s}\right)\right\}
$$

From (3) we build a data map with the same size as the difference image and identical $(x, y)$ locations that those of the pixels in $D$ and nodes in the network. Each node $i$ at location $(x, y)$ is loaded with the data information $r(i)$ according to the following criterion,

$$
r(i)=(-1)^{s+1} P\left(H_{s} / D(x, y)\right) ; \quad s=\{0,1\}
$$

The data consistency between nodes $i, j$ is measured through the data compatibility coefficient $d_{i j}$ as follows,

$$
d_{i j}= \begin{cases}1-0.5|r(i)-r(j)| & j \in N_{i}^{8} \\ 0 & j \notin N_{i}^{8}\end{cases}
$$

$N_{i}^{8}$ is the 8 -connected neighborhood of the node $i$. From (5) we can see that $d_{i j}$ ranges in $[0,1]$ where the lower/higher limit means minimum/maximum data consistency respectively.

The mapping of contextual consistencies is computed through the equation (6) taking into account mutual influences.

$$
c_{i j}= \begin{cases}p_{i j}\left(1-\left|A_{i}^{k}-A_{j}^{k}\right|\right) & j \in N_{i}^{8} \\ 0 & j \notin N_{i}^{8}\end{cases}
$$

where $p_{i j}$ is a coefficient that measures the influence of the node $i$ over node $j$ taking into account the relevance of each node. The relevance is a concept introduced in this paper in order to measure the strength of each node against changes in its activation level. We build an accumulator of cells of size $n=M x N$, where the each cell $i$ is associated to the node of identical name. Each cell $i$ contains the number of times, $p_{i}$, that the node $i$ has changed significantly its activation level. Initially, all $p_{i}$ are set to zero 
and then $p_{i}=p_{i}+1$ if $\left|A_{i}^{k}-A_{i}^{k-1}\right|>\varepsilon$, where $\varepsilon$ is set to 0.05 in this paper. Now, $p_{i j}$ is computed as follows,

$$
p_{i j}= \begin{cases}p_{j} /\left(p_{i}+p_{j}\right) & \left(p_{i}+p_{j}\right) \geq 2 \\ 1 & \text { else }\end{cases}
$$

The equation (7) measures the fraction of changes accumulated for the node $j$ against the node $i ; p_{j i}$ measures the reverse influence of $j$ over $i$. This equation is interpreted as follows, if $p_{i}<p_{j}$ the node $i$ has accumulated less number of changes than the node $j$, i.e. the node $i$ has a higher relevance than the node $j$ and vice versa. This implies that $p_{i j}$ could be different from $p_{j i}$. From (6) we can see that $c_{i j}$ varies with the iteration and ranges in $[0,1]$ where the lower/higher limit means minimum/maximum contextual consistency respectively. One can see that $c_{i j}$ could be different from $c_{j i}$ (non symmetry).

Now the goal is to combine appropriately $d_{i j}$ and $c_{i j}$ in order to derive the causal weights required by the equation (1). Making use of the fuzzy set theory, we consider the fuzzy sets $A$ and $B$, where their elements are pairs of nodes $(i, j)$ and the degrees of compatibility (membership functions) are given by $d_{i j}$ and $c_{i j}$ respectively. According to the dissertations of Zimmermann [10] we propose the Hamacher's union operator because of its performance,

$$
W_{i j}=\frac{(\gamma-1) d_{i j} c_{i j}+d_{i j}+c_{i j}}{1+\gamma c_{i j} d_{i j}}
$$

where $\gamma \geq-1$; by setting $\gamma=-1$ the Hamacher's union operator matches with the Hamacher sum. Hence, in our experiments the best results are obtained with this value.

Once we have computed $W_{i j}$ according to equation (8) a rescaling from the range $[0,+1]$ to the range $[-1,+1]$ allows us to compute the causal weight between nodes $i$ and $j$ as required by the equation (1),

$$
w_{i j}=2 W_{i j}-1
$$

In Tsadiras and Margaritis [5,6] is reported after the experimentation that the decay factor must range in $[0,0.4]$ trying to avoid that the system collapses to zero. This factor subtracts a fraction to the current activation level. Hence, we assume that if a node $i$ is relevant as compared to its neighbors, the fraction to be subtracted must be as minimum as possible and the number of iterations $k$ must be embedded as follows,

$$
d_{i}= \begin{cases}\frac{\bar{p}_{j}}{\left(\bar{p}_{j}+p_{i}\right) k} & k \geq 4 \\ 0 & \text { else }\end{cases}
$$


where $p_{i}$ is the cell's accumulator value for the node $i$ and $\bar{p}_{j}$ is the average value accumulated by the nodes $j \in \boldsymbol{N}_{i}^{8}$.

The network is initialized from the histogram of the difference image $D(x, y)$ according to the method described in Bruzzone and Prieto [3] and reproduced in Pajares [2]. The iterative process ends if all nodes in the network fulfill the following criterion: $\left|A_{i}^{k}-A_{i}^{k-1}\right|>\mathcal{E}$ or a number of iterations, $k_{\max }$ is reached. The final values $A_{i}^{k}$ determine the magnitude of the change.

\section{Performance Analysis}

This paper introduces the relevance criterion when mapping the contextual consistencies, which could introduce a non symmetric influence between two nodes. This implies a modification with respect the HNN approach described in Pajares [2]. So, for comparative purposes we use the same four data sets described in [2]: 1) 40 pairs of real video sequences of outdoor environments of size $1392 \times 1040$; 2) 36 pairs of real video sequences of indoor environments of size $840 \times 760$; 3) 10 pairs of real remote sensing images of size of size $400 x 400$; and 4) 60 pairs of synthetic sensing images of size of size $400 \times 400$. All results are verified against a ground truth map which is previously determined. Figure $1(a, b)$ shows a representative pair of the outdoor environment with the changes detected by the FCM process in $(c)$.

The HNN method of Pajares [2] was compared against six existing image change detection strategies including the approach of Bruzzone and Fernández-Prieto [3] (BRU), which follows the MRF framwork. The best performances were obtained by HNN followed by BRU. This paper compares the performance of the proposed FCM approach against the iterative HNN and BRU methods. FCM, HNN and BRU use the same initialization process and a neighborhood region of size $3 \times 3$. We use a set of seven experiments, a brief description of the experiments is the following: E1: 30 outdoor pairs of images from the same sequence; E2: 10 outdoor pairs of images from different sequences of the same scene; E3: 12 indoor pairs of images from the same sequence without changes in the illumination levels; E4: 12 indoor pairs of images from the same sequence, during the full capture process the illumination levels are online changed, i.e. the images have different intensity levels; E5: 12 indoor pairs of images from the same sequence, an image is obtained without changes in the illumination during its capture and the other, as before, by varying on-line the illumination; E6: 10 pairs of remote sensing images of the same scene; E7: 30 pairs of synthetic remote sensing images of the same scene corrupted with Gaussian noise of zeromean.

The results obtained for each method are compared against the ground truth, based on the PCC magnitude described in Rosin and Ioannidis [11], also used in Pajares [2]: $P C C=(T P+T N) /(T P+F P+T N+F N)$, where TP: number of change pixels correctly detected; FP: number of no-change pixels incorrectly labelled as change; $\mathrm{TN}$ : number of no-change pixels correctly detected; FN: number of change pixels incorrectly labelled as no-change. 


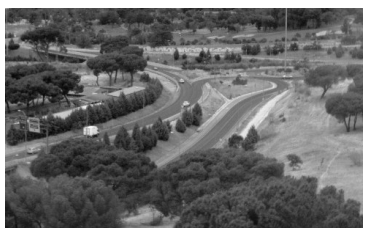

(a)

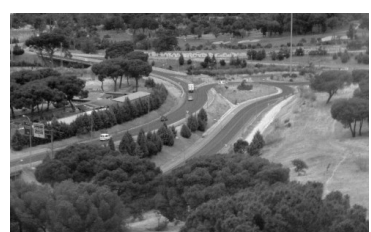

(b)

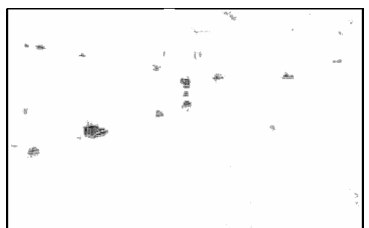

(c)

Fig. 1. Outdoor environment; $(a)$ and $(b)$ two images of the same sequence; $(c)$ changes detected with the FCM approach

Table I shows the results in terms of the correct classification for the seven experiments. The final result for each experiment is averaged by the number of pairs of images processed. The number of iterations used in our FCM $\left(k_{\max }\right)$ is set to the number of iterations where $\mathrm{HNN}$ gained the convergence for each set of experiments, i.e. $\mathrm{E} 1, \mathrm{E} 3=4, \mathrm{E} 2, \mathrm{E} 5=8, \mathrm{E} 4=10$ and $\mathrm{E} 6, \mathrm{E} 7=5$.

Table 1. Averaged PCC scores for each method against the set of experiments

\begin{tabular}{|l|c|c|c|c|c|c|c|}
\hline$x 10^{-3}$ & E1 & E2 & E3 & E4 & E5 & E6 & E7 \\
\hline BRU & 921 & 821 & 945 & 653 & 698 & 819 & 615 \\
\hline HNN & 987 & 943 & 991 & 789 & 876 & 901 & 847 \\
\hline FCM & 944 & 954 & 956 & 823 & 901 & 848 & 844 \\
\hline
\end{tabular}

From the results in Table I, one can see that FCM improves the performance of HNN for experiments E2, E4 and E5 where the number of iterations is higher than the used for the other experiments. This means that the FCM approach is suitable for images where the number of iterations is high.

The above behavior appears when the pair of images displays high variability due to different illumination conditions or other causes as in the experiments E2, E4 and E5. This means that the FCM should be applied in image sequences captured under such illumination conditions where it is foreseeable that the number of iterations could become high.

The best performance achieved by the FCM approach can be interpreted in the light of the mutual influence between two nodes based on the relevance's values. Indeed, as the number of iterations increases, the relevance of each node achieves higher stability (less number of changes in the activation level). This is reflected in the equation (6). FCM and HNN achieve a similar performance for E7 (with noise).

\section{Conclusions}

In this paper we have developed a new automatic strategy for image change detection based on the well-founded FCM paradigm, which allows the computation of a nonsymmetric weight matrix based on binary relations between the nodes in the network thanks to the introduction of the relevance concept. The FCM paradigm also includes unary relations as in HNN. The FCM has proven its performance against some 
existing strategies. As all iterative approaches this method is computational intensive. The execution time is similar to the obtained by HNN. So, for real-time requirements under surveillance tasks it should be implemented under parallel architectures.

Acknowledgments. This research was sponsored under project no. 143/2004 Fundación General UCM and the Spanish Council for Science and Technology (CICYT) under grant DPI2006-15661-C02-01.

\section{References}

1. Radke, R.J., Andra, S., Al-Kofahi, O., Roysam, B.: Image Change Detection Algorithms: A Systematic Survey. IEEE Trans. Image Processing 14(3), 294-307 (2005)

2. Pajares, G.: A Hopfield Neural Network for Image Change Detection. IEEE Trans. Neural Networks 17(5), 1250-1264 (2006)

3. Bruzzone, L., Fernández-Prieto, D.: Automatic Analysis of the difference Image for unsupervised change detection. IEEE Trans. Geoscience Remote Sensing 38(3), 1171-1182 (2000)

4. Aach, T., Kaup, A.: Bayesian algorithms for adaptive change detection in image sequences using Markov Random fields. Signal Processing: Image Communication 7, 147-160 (1995)

5. Tsardias, A.K., Margaritis, K.G.: An experimental study of the dynamics of the certainty neuron fuzzy cognitive maps. Neurocomputing 24, 95-116 (1999)

6. Tsardias, A.K., Margaritis, K.G.: Cognitive Mapping and Certainty Neuron Fuzzy Cognitive Maps. Information Sciences 101, 109-130 (1997)

7. Kosko, B.: Fuzzy Cognitive Maps. Int. J. Man. Machine Studies 24, 65-75 (1986)

8. Kosko, B.: Neural Networks and Fuzzy Systems: a dynamical systems approach to machine intelligence. Prentice-Hall, NJ (1992)

9. Miao, Y., Liu, Z.Q.: On Causal Inference in Fuzzy Cognitive Maps. IEEE Trans. Fuzzy Systems 8(1), 107-119 (2000)

10. Zimmermann, H.J.: Fuzzy Set Theory and its applications. Kluwer Academic Publishers, Dordrecht (1991)

11. Rosin, P.L., Ioannidis, E.: Evaluation of global image thresholding for change detection. Pattern Recognition Letters 24, 2345-2356 (2003) 